University of Wollongong

Research Online

Faculty of Engineering and Information

Faculty of Engineering and Information

Sciences - Papers: Part A

Sciences

2013

Fabrication and characterization of PDMS based magnetorheological elastomers

Weihua Li

University of Wollongong, weihuali@uow.edu.au

Masami Nakano

Tohoku University

Follow this and additional works at: https://ro.uow.edu.au/eispapers

Part of the Engineering Commons, and the Science and Technology Studies Commons

Research Online is the open access institutional repository for the University of Wollongong. For further information contact the UOW Library: research-pubs@uow.edu.au 


\title{
Fabrication and characterization of PDMS based magnetorheological elastomers
}

\begin{abstract}
This paper presents the fabrication and characterization of a new magnetorheological elastomer (MRE) by using polydimethylsiloxane (PDMS) as a matrix. The base and curing agent of PDMS with a weight ratio of 10:1 were mixed first as the carrying matrix, and then carbonyl iron particles were added to the matrix and stirred sufficiently. The final mixture was placed in a vacuum chamber to eliminate bubbles for $30 \mathrm{~min}$ and was moulded later to form membranes of $1 \mathrm{~mm}$ thickness. A total of four PDMS based MRE samples, with different weight fractions of $60 \%, 70 \%, 80 \%$, and $90 \%$, were fabricated. Their mechanical properties under both steady-state and dynamic loading conditions were tested. The effects of particle composition, magnetic field, strain amplitude and frequency on the MRE effects were summarized. With the increase of iron particle composition, the magnetorheological effects of the samples increase steadily. It is also noted that the initial modulus of the MRE samples shows an increasing trend with the iron particle composition. Additionally, the microstructures of the PDMS based MREs were also observed by a low vacuum scanning electric microscope (LV-SEM).
\end{abstract}

\section{Keywords}

fabrication, characterization, pdms, magnetorheological, elastomers

Disciplines

Engineering | Science and Technology Studies

\section{Publication Details}

Li, W. \& Nakano, M. (2013). Fabrication and characterization of PDMS based magnetorheological elastomers. Smart Materials and Structures, 22 (5), 055035-1-055035-7. 


\title{
Fabrication and Characterisation of PDMS based Magnetorheological Elastomers
}

\author{
W.H. Li ${ }^{* 1}$, and M. Nakano ${ }^{2}$ \\ ${ }^{1}$ School of Mechanical, Materials and Mechatronic Engineering, University of Wollongong, \\ Wollongong, NSW 2522, Australia \\ ${ }^{2}$ Intelligent Fluid Control Laboratory, Institute of Fluid Science, Tohoku University, 2-1-1 Katahira, \\ Aoba-ku, Sendai, 980-8577, Japan
}

\begin{abstract}
This paper presents the fabrication and characterisation of a new magnetorheological elastomer (MRE) by using Polydimethylsiloxane (PDMS) as a matrix. The base and curing agent of PDMS with a weight ratio of 10:1 were mixed first as the carrying matrix, then carbonyl iron particles were added to the matrix and stirred sufficiently. The final mixture was placed in a vacuum chamber to eliminate bubbles in 30 minutes and was molded later to be membranes at $1 \mathrm{~mm}$ thickness. A total of four PDMS based MRE samples, with different weight fractions of $60 \%, 70 \%, 80 \%$, and $90 \%$, were fabricated. Their mechanical properties under both steady-state and dynamic loading conditions were tested. The effects of particle composition, magnetic field, strain amplitude and frequency on the MRE effects were summarized. With the increase of iron particle composition, the PDMS based MRE samples increase steadily. It is also noted that the initial modulus of the MRE samples shows an increasing trend with the iron particle composition. Additionally, the microstructures of the PDMS based MREs were also observed by a low vacuum scanning electric microscope (LV SEM).
\end{abstract}

Keywords: MR elastomers, PDMS, shear modulus.

\footnotetext{
*Corresponding author: weihuali@uow.edu.au
} 


\section{Introduction}

Magnetorheological (MR) materials, including MR fluids, MR elastomers and MR foams, are smart materials that their mechanical and/or rheological properties can be controlled by external magnetic fields [1-4]. As an important family member of MR materials, MR elastomers are composites that polarized particles are suspended in a non-magnetic solid or gel-like matrix. Different from MR fluids whose viscosity varies with magnetic field, MR elastomers exhibit characteristics that their moduli can be reversely controlled by an external magnetic field. MR elastomers have recently found a variety of applications, such as adaptive tuned vibration absorbers, dampers, sensors and so on [5-10].

MREs generally consist of three major components: magnetizable particles, matrix and additives. Iron particles are generally used as the filler material to fabricate MREs. Both natural rubber and silicone rubber are used as typical matrixes [11,12]. Additives are commonly used to adjust the mechanical and chemical properties or electrical performance of MR fluids [13] as well as MR elastomers [14]. Silicone oil is an additive to increase the gaps between the matrix molecules and to decrease the gaps between the conglutination of molecules. Apart from increasing the plasticity and fluidity of the matrix, the additives can average the distribution of internal stress in the materials, which makes them ideal for fabricating MREs [15].

Natural rubber is an elastomer. The purified form of natural rubber is the chemical polyisoprene which can also be produced synthetically. Heat is normally required to vulcanise silicone rubber. The silicone rubber and a vulcanising silicon sealant (at room temperature) are mixed with silicon oil to changing its ductility. The silicon oil is selected on the basis of preliminary studies with different elastomers. Polydimethylsiloxanes (PDMS) is one example of silicon rubber. The PDMS have a low surface tension and are capable of wetting most surfaces. The stability and chemical neutrality of the system also enables the adhesive to bond to the metals [16]. PDMS is a widely used silicon-based organic polymer, and is particularly known for its unique properties such as curing at low temperatures, rapid curing 
at high temperature, deformation reversibility and surface chemistry controllable by reasonably well-developed techniques. PDMS is optically clear, and, in general, is considered to be inert, non-toxic and non-flammable $[17,18]$. Previous studies have mainly focused on the structural properties of PDMS [19] and the kinetic properties under crystallization from solution [20]. In our previous work, we did pioneering work in using PDMS a matrix for fabricating and characterising patterned MREs with precise BCT and BCC microstructures [21]. However, the fabrication steps were different from conventional MRE fabrication methods as the PDMS was simply filled in the mould to mix with iron particles which were already positioned in the mould with the designed microstructures. It is noted that the methods reported in [21] is different from the fabrication method in this work. In this study, the PDMS will work as a new matrix. Its unique temperature dependent curing characteristic will be significant in shortening the fabrication time.

This paper is organized as follows. Followed by the Introduction, the fabrication of isotropic PDMS MRE samples were described. The SEM images of these samples will be reported in the following Section. Section 3 presents the characterization of both steady and dynamic properties of these samples. The main finding will be summarized in the conclusion section.

\section{Fabrication and microstructure observation of PDMS MREs}

\subsection{Materials and fabrication}

The base and curing agent of PDMS, type Sylgard 184 (Dow Corning Pty. LTD) at a weight ratio 10:1 were firstly mixed evenly to be used as the carrying matrix, and then added to the carbonyl iron particles, type C3518 (Sigma-Aldrich Pty. LTD) and stirred sufficiently. The iron particles' diameter is between $3 \mu \mathrm{m}$ and $5 \mu \mathrm{m}$ at a normal distribution. The density of iron particles and PMDS are $7.86 \mathrm{~g} / \mathrm{cm}^{3}$ and $1.1 \mathrm{~g} / \mathrm{cm}^{3}$, respectively. The final mixture was placed in a vacuum chamber to eliminate bubbles in 30 minutes. It was then placed between two pieces of plastic paper and pressed to be membranes. A few mall cylinders with a $1 \mathrm{~mm}$ thickness were used to ensure the thickness of MRE samples. The mixture was curing in an oven at $100^{\circ} \mathrm{C}$ for 35 minutes and then cut by a punch to disks with a $20 \mathrm{~mm}$ diameter. The total fabrication such PDMS based MRE sample was less than 100 minutes, which is much 
shorter than that of fabricating conventional MREs made of natural rubber or silicone rubber that generally need more than 24 hours to curing.

In this study, four PDMS MRE samples were fabricated whose iron particles' weight fraction (iron wt. \%) were $60 \%, 70 \%, 80 \%$ and 90\%. All MRE samples were isotropic.

\subsection{Microstructure observation}

A Low Vacuum Scanning Electron Microscope (LV-SEM) (model: JSM 6490) was used to observe the microstructure of PDMS MREs. Figure 1 shows the surface imaging for all MRE samples' microstructures.
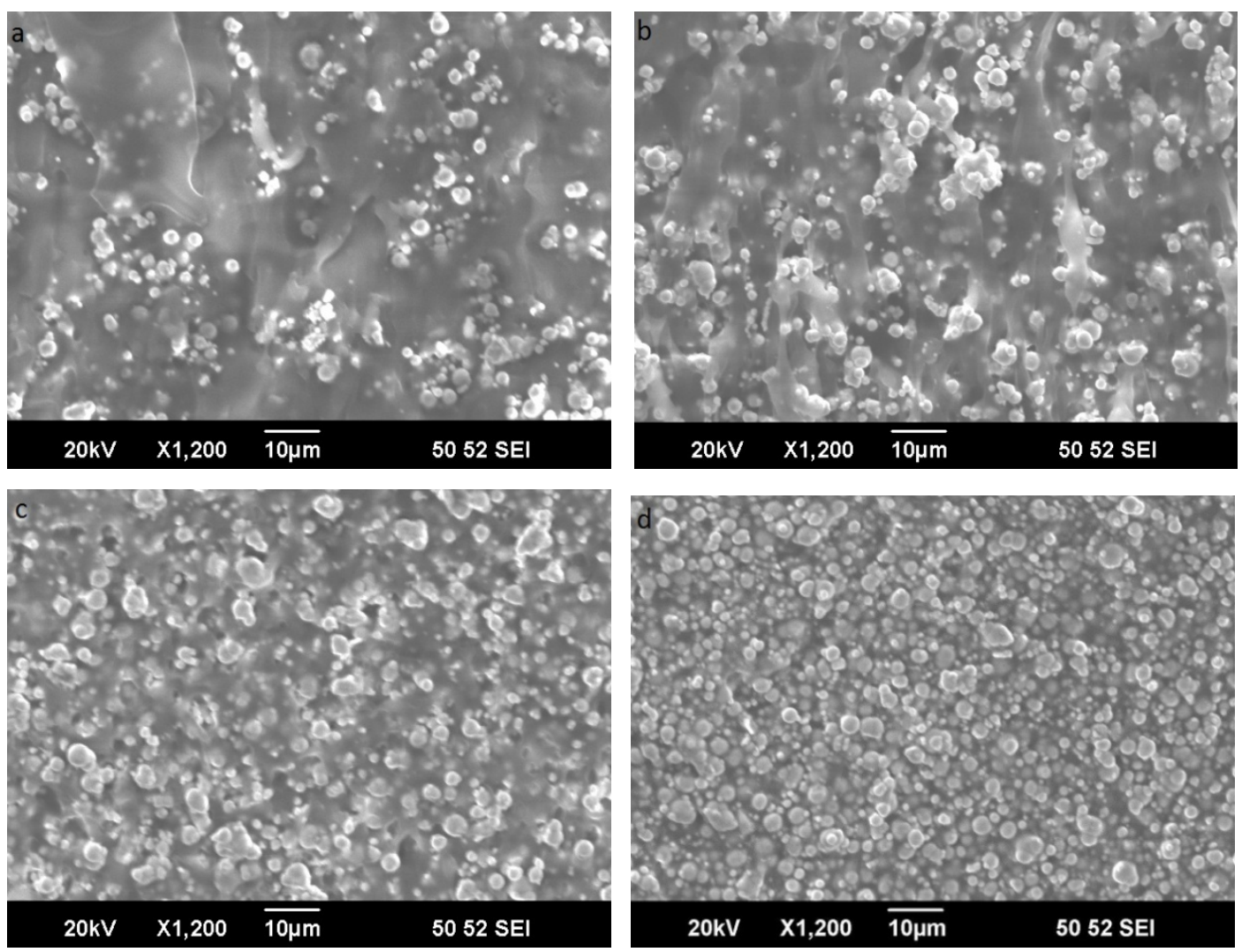

Fig. 1 Microstructure of PDMS MREs a iron $60 \% \mathbf{b}$ iron $70 \% \mathbf{c}$ iron $80 \% \mathbf{d}$ iron $90 \%$

Figures 1a-d shows that the carbonyl iron particles disperse randomly in the PDMS matrix due to the isotropy of all samples. The sample having higher iron weight fraction shows more iron particles in the same area. 


\section{Rheological measurement and results}

The shear-strain dependent rheology of the MREs was measured by a parallel-plate rheometer (MCR 301, Anton Paar Companies, Germany). A Magneto Rheolgical Device (MRD 180, Anton Paar Companies, Germany) was used to generate magnetic field in the test area and a temperature control device (Viscotherm VT2, Anton Paar Companies, Germany) was used to control the measuring sample to be at $25^{\circ} \mathrm{C}$. Steady-state shear strain sweeps, dynamic strain sweeps, dynamic frequency sweeps and magnetic field intensity sweeps were carried out using the PP-20 measuring geometry (20 mm diameter) with a gap of $1 \mathrm{~mm}$. The testing procedure for each measurement is illustrated below. Initially, the sample is sheared at a constant shear rate of $100 \mathrm{~s}^{-1}$ at a zero field for half a minute to distribute the particles uniformly. Next, the desired magnetic field is applied and maintained for more than 30 seconds so that the testing sample forms a static structure. After that, a variety of shear modes, including the steady shear, the dynamic oscillatory shear, and the magnetic field intensity sweeps were employed to measure rheological properties of the samples under steady and dynamic loading conditions.

In this experiment, the magnetic flux density of the sample of MRE (BMRE) in the measuring gap depends not only on the current (I) applied to the samples and the magnetic properties of MRE materials. As the permeability of MRE samples varies little, an empirical equation, $\mathrm{BMRE}=220 \mathrm{I}$, was employed to predict the flux density for different MRE samples, where the units of BMRE and I are in $\mathrm{mT}$ and amp (A), respectively. In the following test, the test current varies from 0 to $2 \mathrm{~A}$ with the increment $0.5 \mathrm{~A}$, whose intensity of magnetic field is 0 to $440 \mathrm{mT}$ with the increment $110 \mathrm{mT}$.

\subsection{Steady state}

Under the rotary shear the shear stress and strain of MREs under fields varying from $0 \sim 440$ $\mathrm{mT}$ were measured at $25^{\circ} \mathrm{C}$ and $5 \mathrm{rad} / \mathrm{s}$ angular frequency. The MR effect was evaluated by 
measuring the shear strain-stress curve of the sample with and without a magnetic field applied. Figures $2 \mathrm{a}-\mathrm{d}$ show the strain-stress curve of different samples at five different magnetic field intensities ranging from 0 to $440 \mathrm{mT}$. The slope of the strain-stress curve is the shear modulus of the material.
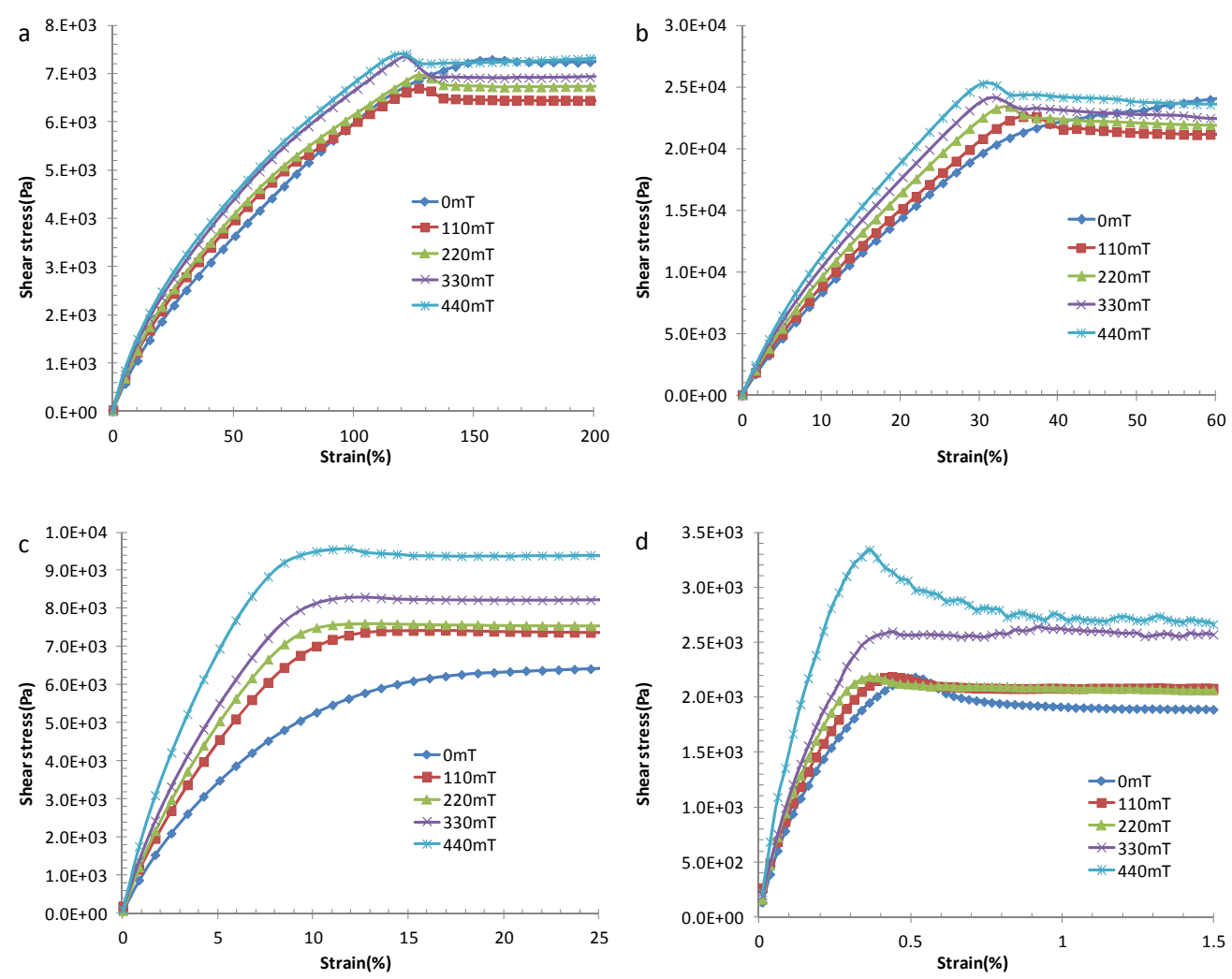

Fig. 2 Strain-stress curve versus magnetic field a iron $60 \%$ b iron $70 \%$ c iron $80 \%$ d iron $90 \%$

As can be seen in the figures, all the samples' shear modulus show an increasing trend with magnetic field before they reach magnetic saturation at high field strength, which proves that all the MRE samples exhibit obvious MR effects. In Fig. 2d, we can see that the curve at $0 \mathrm{mT}$, which is the zero-field modulus of the $90 \mathrm{wt} . \%$ sample, shows a bigger slop of stress-strain curve compared with the lower iron concentration samples, which means 90wt.\% sample has a higher zero-field modulus. When the strain is above the limitation, the shear stress reaches a saturation (maximum value) and decrease steadily. This might be due to the sliding effect. Additionally, other factors, such as the sample surface roughness and the normal force, could contribute to the resultant stress. In particular, they influence the static friction between the MRE sample and the upper plate, which consequently result in overshoots, as shown in Figs. 
2a-d.

Also from Figs. 2a-d, the shear stress shows a linear relationship with the shear strain when the strain is within a certain range. This means the MRE acts with linear viscoelastic properties when the strain is below a limitation. Table 1 summarized the linear ranges of all samples at various magnetic field intensities.

Table 1 Linear range of all samples at different magnetic field

\begin{tabular}{c|c|c|c|c|c}
\hline \multirow{2}{*}{ Samples } & \multicolumn{5}{c}{ Linear range (\%) } \\
\cline { 2 - 6 } & $\mathbf{0 m T}$ & $\mathbf{1 1 0 \mathbf { m T }}$ & $\mathbf{2 2 0 \mathbf { m T }}$ & $\mathbf{3 3 0 \mathbf { m T }}$ & $\mathbf{4 4 0 \mathbf { m T }}$ \\
\hline $60 \%$ iron & 150 & 127 & 127 & 123 & 120 \\
\hline $70 \%$ iron & 80 & 37 & 33 & 31 & 30 \\
\hline $80 \%$ iron & 40 & 10 & 9 & 9 & 8 \\
\hline $90 \%$ iron & 0.5 & 0.4 & 0.35 & 0.35 & 0.35 \\
\hline
\end{tabular}

When the carbonyl iron weight fraction increases from $60 \%$ to $90 \%$, the range of linearity decreases from $120 \%-150 \%$ to $0.35 \%-0.5 \%$. The higher iron particles sample leads to the lower linear range. From these figures it is also noted that the linear visocoelastic range shows a slightly decreasing trend with the magnetic field.

\subsection{Dynamic tests result}

In order to obtain the dynamic mechanical behaviour of MRE, both strain amplitude sweep tests and angular frequency sweep tests were used. Five sets of data were collected for different amplitudes of oscillation, according to the various magnetic field inputs to the samples of MREs. The amplitude of shear strain in angular frequency sweep tests is set at $1 \%$ and the input frequency was $5 \mathrm{~Hz}$ in the strain amplitude sweep tests.

Strain amplitude sweep 
In the strain sweep test, the storage and loss moduli were tested by varying strain from $0.1 \%$ to $100 \%$ at five different magnetic fields ranging from $0 \mathrm{mT}$ to $440 \mathrm{mT}$ and room temperature. Figures 3a-d show the changing of storage modulus and loss moduli at the strain amplitude sweep.
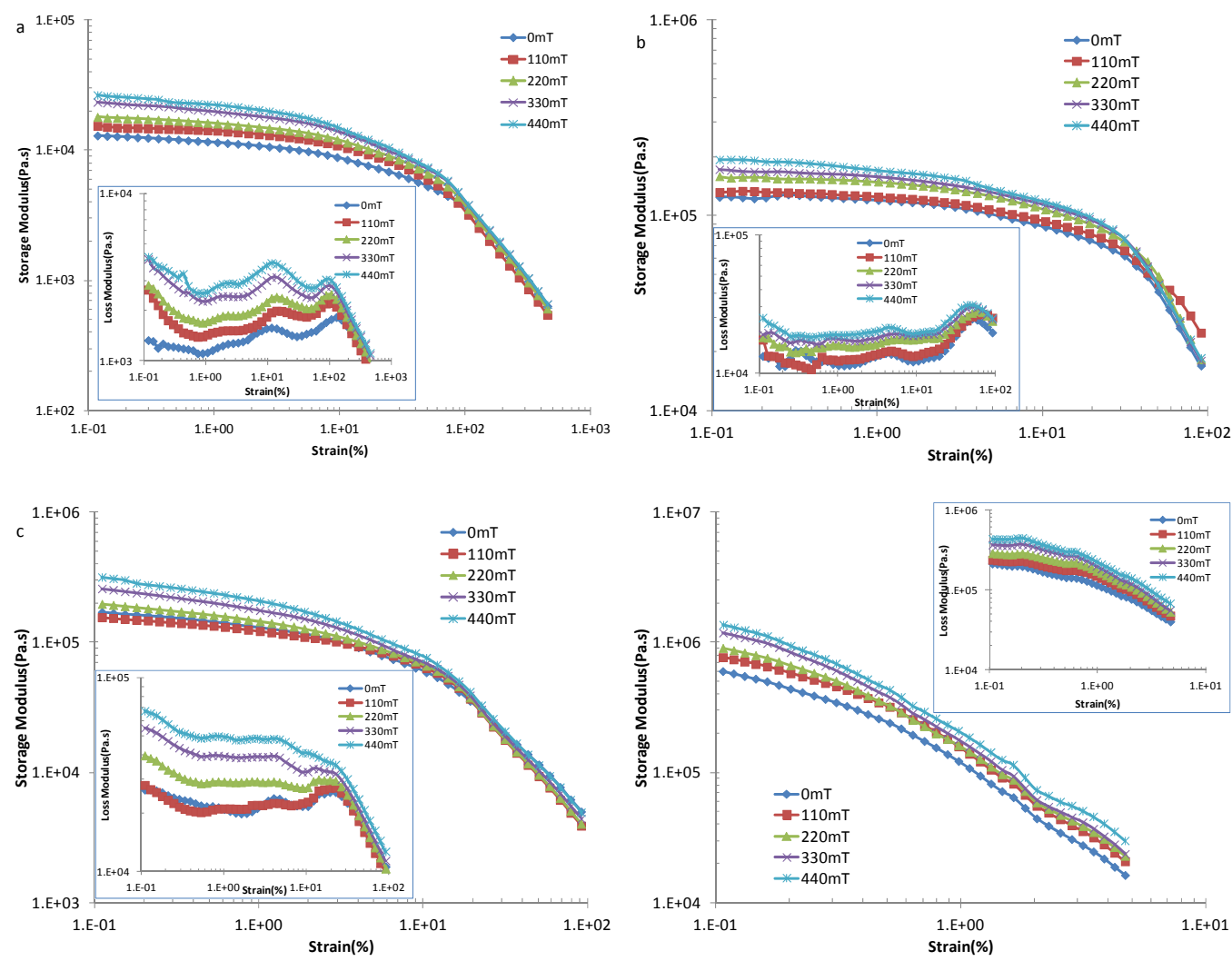

Fig. 3 Storage and Loss Modulus versus strain amplitude sweep a iron $60 \% \mathbf{b}$ iron $70 \% \mathbf{c}$ iron $80 \% \mathbf{d}$ iron $90 \%$

In Figs. 3a-d, the overall trend of storage modulus is decreasing with the strain amplitude. It goes down smoothly within a critical shear strain and begins to drop dramatically over the critical value, which could be used to mark the transition from a linear to nonlinear range, similar to MR fluids [22]. Take the sample containing $80 \%$ iron as an example, within $10 \%$ strain, its storage modulus versus strain as linear.

These figures also show that the storage modulus and loss modulus have a crossing point. Before the crossing point, the storage modulus is higher than the loss modulus, which means that MRE exhibits more solid status. After the crossing, loss modulus start to prevail over the 
storage modulus and a liquid state shows up. Table 2 summarized the crossing points of all samples at various magnetic field intensities. 
Table 2 Crossing points of all samples at different magnetic field

\begin{tabular}{l|c|c|c|c|c}
\hline \multirow{2}{*}{ Samples } & \multicolumn{5}{|c}{ Crossing point (\%) } \\
\cline { 2 - 6 } & $0 \mathrm{mT}$ & $110 \mathrm{mT}$ & $220 \mathrm{mT}$ & $330 \mathrm{mT}$ & $440 \mathrm{mT}$ \\
\hline $60 \%$ iron & 220 & 180 & 180 & 155 & 155 \\
\hline $70 \%$ iron & 78 & 69 & 67 & 67 & 67 \\
\hline $80 \%$ iron & 26 & 24 & 23 & 22 & 21 \\
\hline $90 \%$ iron & 1.05 & 1 & 0.8 & 0.8 & 0.8 \\
\hline
\end{tabular}

From table 2 we can see that the sample with higher iron weight fraction shows a diminishing trend when either iron particles concentration or magnetic field increases.

\section{Angular frequency sweep}

For each test, the strain is set a constant of $1 \%$ while the angular frequency was varied from 1 to $100 \mathrm{1} / \mathrm{s}$ at different magnetic fields of $0,110,220,330$ and $440 \mathrm{mT}$. Figs. 4a-d show the storage and loss moduli curves of the MRE samples under frequency sweep and at $25^{\circ} \mathrm{C}$.
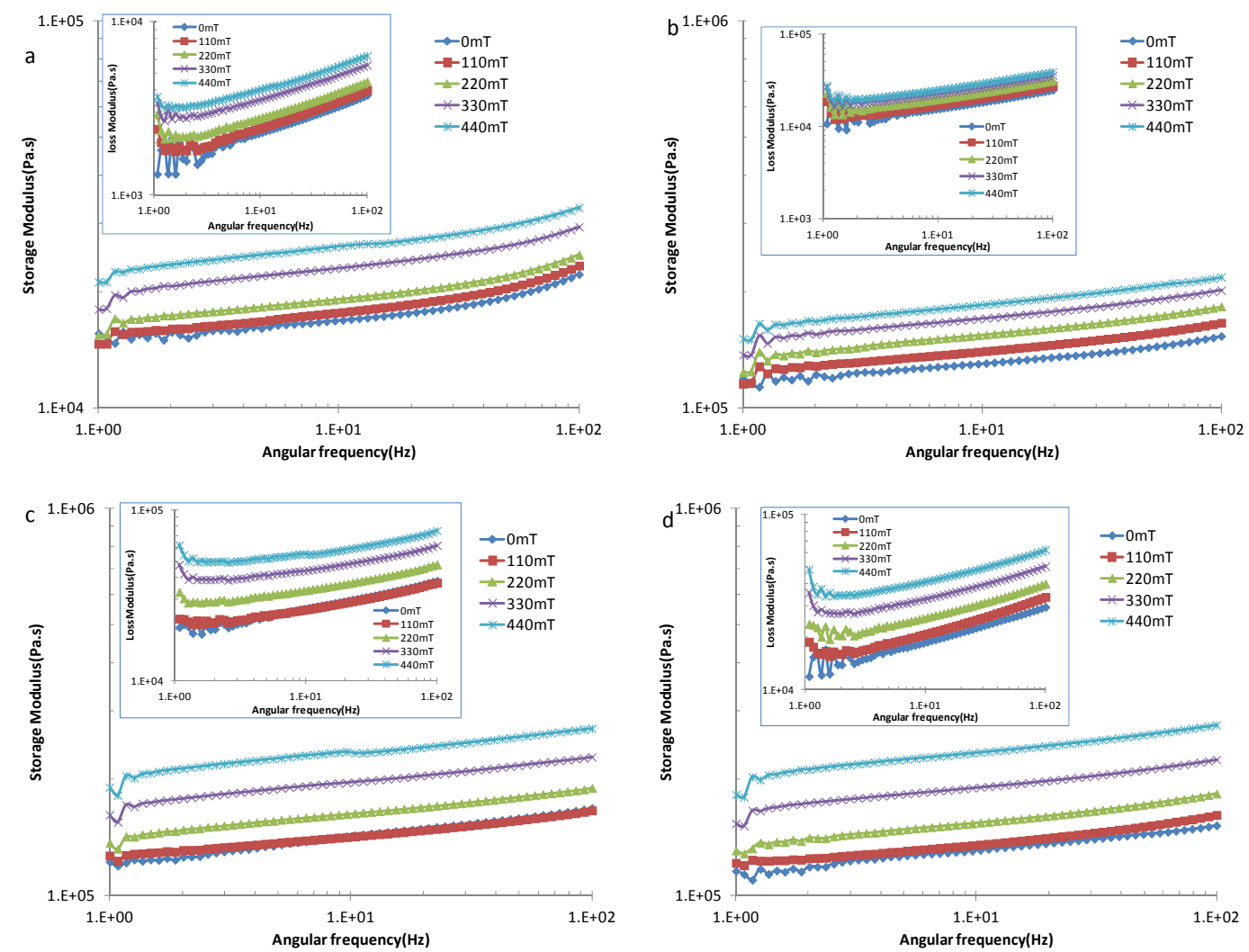
Fig. 4 Storage and Loss Modulus versus angular frequency sweep a iron $60 \% \mathbf{b}$ iron $70 \% \mathbf{c}$ iron $80 \% \mathbf{d}$ iron $90 \%$

From the figures above, we can see that in the log-log scale, the storage and loss moduli of all the samples are both increasing linearly with the growth of angular frequency. This logarithmically linear relationship of the storage and loss moduli to the angular frequency could be used to predict the storage and loss moduli at a certain frequency.
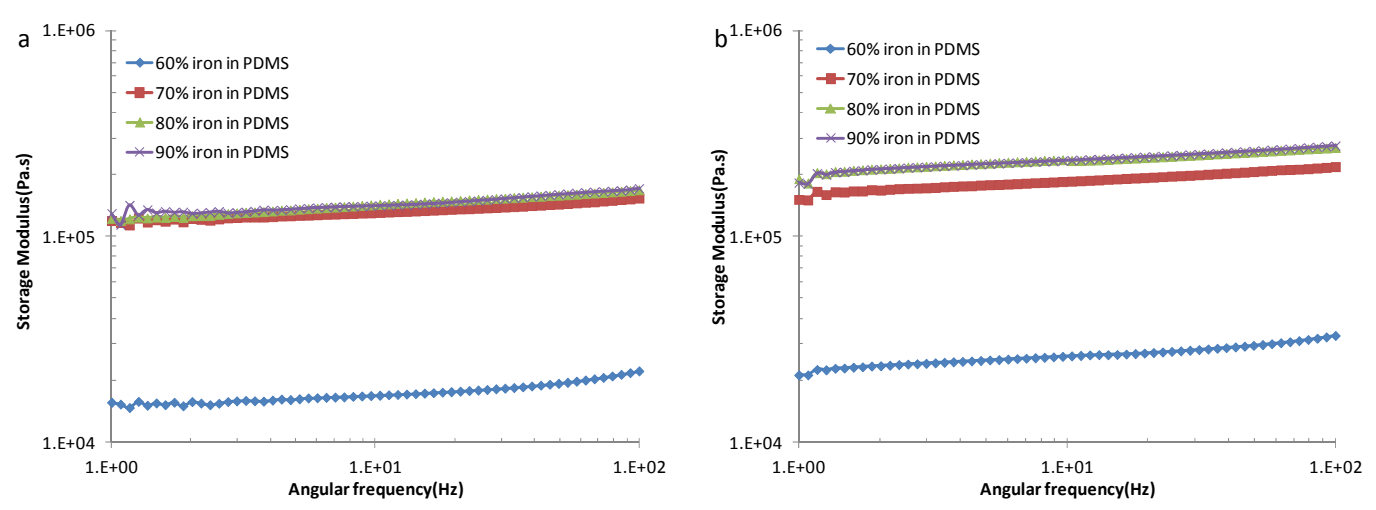

Fig. 5 Storage Modulus versus angular frequency sweep of different samples a $0 \mathrm{mT}$ b $440 \mathrm{mT}$

The effect of the iron weight fraction on the MR effect was shown in Figs. 5a-b. It can be seen from these figures that $60 \%$ iron sample has the lowest storage modulus, but the other three samples show very similar storage modulus at both low and high magnetic fields. This implies that the overall initial modulus of the MRE samples depends mainly on the PDMS matrix when the iron particle's weight fraction value is below $70 \%$. However, when the weight fraction is above $70 \%$, the initial modulus increases sharply, which means that the iron particle contribute largely to the overall stiffness of the MRE samples.

\subsection{Magnetic field intensity sweep}

The strain and angular frequency are set at $1 \%$ and $1 \mathrm{rad} / \mathrm{s}$ respectively in this test. Magnetic field intensity is increasing from $0 \mathrm{mT}$ to $440 \mathrm{mT}$ linearly. This test is mainly used to figure out the relative MR effect $\left(\mathrm{G}^{\prime}{ }_{\max } / \mathrm{G}^{\prime}{ }_{0}\right)$ of each sample. $\mathrm{G}_{0}{ }_{0}$ denotes the MRE samples' zero-field storage modulus, $G^{\prime}{ }_{\max }$ denotes the saturated field-induced modulus, and $G^{\prime}{ }_{\max } / G^{\prime}{ }_{0}$ denotes 
the relative MR effect. Figs. 6a-b show the storage modulus curves of the MRE samples versus magnetic field intensity at $25^{\circ} \mathrm{C}$.
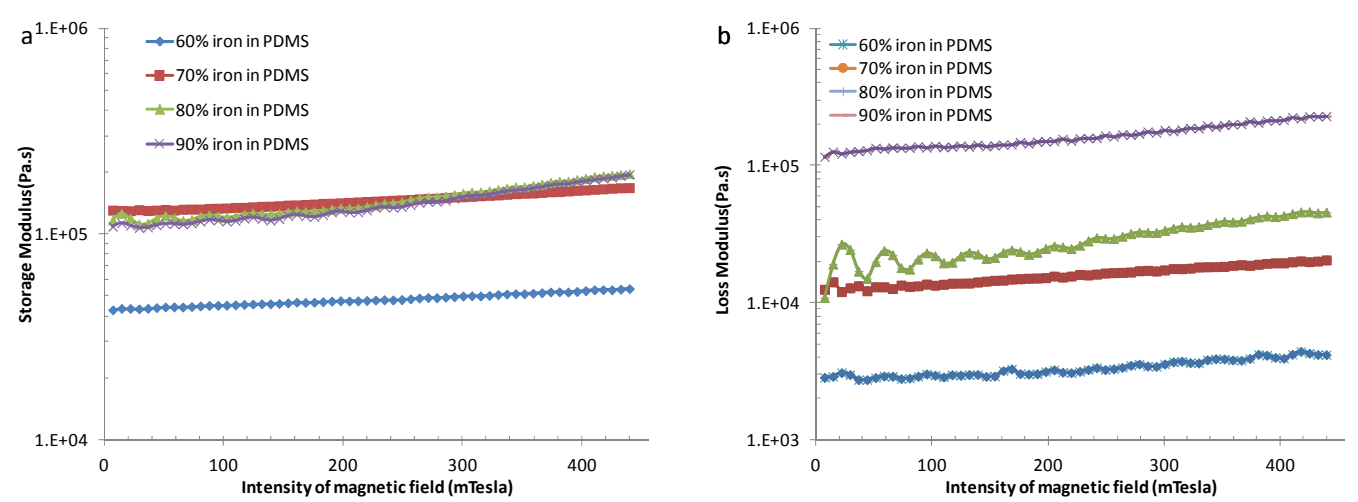

Fig. 6 Storage and loss Moduli versus magnetic field intensity of different samples a Storage modulus b Loss modulus

From Figs. 6a we can see that the relative MR effect is $1.27,1.30,1.74$ and 1.81 for $60 \%$, $70 \%, 80 \%$ and $90 \%$ samples, respectively. The sample with higher iron particle concentration has higher relative MR effect.

\section{Conclusion}

Four isotropic PDMS based MRE sample with 60\%-90\% iron particles weight fractions were fabricated in this study. LV SEM was used to observe their microstructures. This observation shows that iron particles disperse randomly in the isotropic samples.

The magnetic field intensity sweep, steady state and dynamic tests such as strain amplitude sweep and angular frequency sweep were used to test the magnetorheology of PDMS MREs. Because of the strong mechanical properties of PDMS, both the storage and loss moduli have smaller changes than conventional MREs. The steady state tests showed that the increase of iron particles in the sample would diminish the viscoelastic linear range of MREs. The dynamic and magnetic field intensity sweep test proved that the samples with higher iron weight fraction show higher initial storage and loss moduli and also higher MR effects. 


\section{Acknowledgements}

This work is supported by the "General Collaborative Research Project 2012" scheme, IFS, Tohoku University.

\section{References}

1. Carlson JD, Jolly MR (2000) MR fluid, foam and elastomer devices. Mechatronics 10 555-569.

2. Park BJ, Fang FF, Choi HJ (2010) Magnetorheology: materials and applications. Soft Matter 6(21): 5246-5253.

3. Wang DH, Liao WH (2011) Magnetorheological fluid dampers: a review of parametric modelling. Smart Mater Struct 20 (2): 023001.

4. Ginder JM, Clark SM, Schlotter WF, Nichols ME (2002) Magnetostrictive phenomena in magnetorheological elastomers. Int J Mod Phys B 16(17\&18):2412-2418.

5. Deng HX, Gong XL, Wang LH (2006) Development of an adaptive tuned vibration absorber with magnetorheological elastomer. Smart Mater Struc 15(5):N111-N116.

6. Albanese AL, Cunefare KA (2008) Performance of MRE-based vibration absorbers. J Intell Mater Syst Struct 19: 551-563.

7. Zhang XZ, Li WH (2009) Adaptive tuned dynamic vibration absorbers working with MR elastomers. Smart Struct Syst 5(5):517-529.

8. Ni ZC, Gong XL, Li JF, Chen L (2009) Study on a dynamic stiffness-tuning absorber with squeeze-strain enhanced magnetorheological elastomer. J Intell Mater Syst Struct 20(10): 1195-1202.

9. Bica I (2007) Giant resistances based on magnetorheological suspensions. J Ind Eng Chem 13 (2): 299-304.

10. Wang X, Chafoorianfar N, Gordaninejad F (2011) Study of electrical conductivity in magnetorheological elastomers. In: 2011SPIE International Conference on Active and Passive Smart Structures and Integrated Systems, Proceedings of SPIE, Vol. 7977, Art. No: 797710.

11. Gong XL, Zhang XZ, Zhang PQ (2005) Fabrication and characterisation of isotropic magnetorheological elastomers. Polymer Test 24 (5): 669-676.

12. Li WH, Zhou Y, Tian TF (2010) Viscoelastic properties of MR elastomers under harmonic loading. Rheol Acta 49:733-740.

13. Fang FF, Choi HJ, Jhon MS (2009) Magnetorheology of soft magnetic carbonyl iron suspension with single-walled carbon nanotube additive and its yield stress scaling function. Colloids Surf A 351:46-51. 
14. Tian TF, Li WH, Deng YM (2011) Study of sensing capabilities of MR elastomers. Smart Mater Struct 20:025022.

15. Leblanc JL (2002) Rubber-filler interactions and rheological properties in filled compounds. Prog Polym Sci 27(4):627-687.

16. De Buyl F (2001) Silicone sealants and structural adhesives. Int J Adhes Adhes 21:411-422.

17. McDonald JC, Duffy DC, Anderson JR, Chiu DT, Wu HK, Schueller OJA, Whitesides $\mathrm{G}$ (2000) Fabrication of microfluidic systems in poly(dimethylsiloxane). Electrophoresis 21(1): 27-40.

18. Noonan KJT, Gates DP (2008) Inorganic and organometallic polymers. Annual Reports Section "A" (Inorganic Chemistry) 104: 394-413.

19. Rajan GS, Sur GS, Mark JE, Schaefer DW, Beaucage G (2003). Preparation and characterization of some unusually transparent poly(dimethylsiloxane) nanocomposites. J Polym Sci Part B: Polym Phys 41(16): 1897-1901.

20. Feio G, Buntinx G, Cohen-Addad JP (1989). NMR Approach to the kinetics of polymer crystallization .2. polydimethylsiloxane solutions. J Polym Sci Part B Polym Phys 27(1): 1-24.

21. Zhang XZ, Peng SL, Wen WJ, Li WH (2008) Analysis and fabrication of patterned magnetorheological elastomers. Smart Mater Struct 17(4):045001.

22. Li WH, Du H, Chen G, Yeo SH, Guo NQ (2003) Nonlinear Viscoelastic Properties of MR Fluids under Large-Amplitude Oscillatory Shear. Rheol. Acta, 42 (3): 280-286 